\title{
Upregulation of microRNA-140-3p mediates dachshund family transcription factor 1 expression in immunoglobulin $A$ nephropathy through cell cycle-dependent mechanisms
}

\author{
XIAOBIN ZHOU ${ }^{1}$, YAO LU ${ }^{2}$, PENGFEI GUO ${ }^{3}$ and CHENGLIN ZHOU ${ }^{1}$ \\ ${ }^{1}$ Department of Clinical Laboratory, Taizhou People's Hospital, Taizhou, Jiangsu 225300; \\ ${ }^{2}$ Department of Teaching Research of Medical Technology, Gannan Medical University, Ganzhou, Jiangxi 341000; \\ ${ }^{3}$ Department of Clinical Laboratory, Shanghai Tenth People's Hospital, Shanghai 200072, P.R. China
}

Received May 29, 2020; Accepted November 11, 2020

DOI: $10.3892 / \mathrm{mmr} .2020 .11773$

\begin{abstract}
Immunoglobulin A nephropathy (IgAN) is a kidney disease and one of the commonest forms of glomerulonephritis worldwide. The present study investigated the role of dachshund family transcription factor 1 (DACH1) in IgAN and identified one of its binding microRNAs (miRNAs). The expression of DACH1 in human mesangial cells (HMCs) incubated with polymeric $\operatorname{IgA}(\mathrm{pIgA})$ isolated and purified from the serum of patients with IgAN or healthy individuals was evaluated by reverse transcription-quantitative (RT-q) PCR and western blotting. Cell proliferation and cell cycle assays were performed in DACH1-overexpressing HMCs to identify the role of DACH1 in IgAN and enzyme-linked immunosorbent assay was carried out to verify the release of inflammatory factors from HMCs. The target miRNAs of DACH1 were predicted using bioinformatics software and miR-140-3p was identified as a target of DACH1 by luciferase report assay, RT-qPCR and western blotting. The results demonstrated that DACH1 was downregulated in HMCs cultured with $\mathrm{pIgA}-\mathrm{IgAN}$ at both mRNA and protein levels. Overexpression of DACH1 suppressed HMC growth and inhibited inflammatory cytokine release from HMCs cultured with $\mathrm{pIgA}-\mathrm{IgAN}$. The expression of DACH1 was negatively regulated by miR-140-3p in IgAN and miR-140-3p inhibition suppressed HMC growth and inhibited inflammatory cytokine
\end{abstract}

Correspondence to: Dr Chenglin Zhou, Department of Clinical Laboratory, Taizhou People's Hospital, 366 Taihu Road, Taizhou, Jiangsu 225300, P.R. China

E-mail: jszhoucl@126.com

Abbreviations: IgAN, immunoglobulin A nephropathy; DACH1, dachshund family transcription factor 1; HMCs, human mesangial cells; pIgA, polymeric IgA; 3'UTR, 3'-untranslated region; CXCL1, chemokine cc-motif ligand 1; NC, negative control.

Key words: dachshund family transcription factor 1, human mesangial cells, cell differentiation, cell cycle, miR-140-3p, immunoglobulin A nephropathy release from HMCs cultured with pIgA-IgAN. The findings of the present study demonstrated that DACH1 decreased HMC growth and the release of inflammatory cytokines from HMCs may be targeted by miR-140-3p. The results suggested that DACH1 could be associated with the progression of IgAN and provide a potential target for further studies related to the mechanism of $\operatorname{Ig} \mathrm{AN}$.

\section{Introduction}

Immunoglobulin A nephropathy (IgAN) is an immune complex glomerulonephritis characterized by IgA deposition in the mesangial area, abnormal proliferation of mesangial cells and excessive expansion of the mesangial matrix $(1,2)$. It is one of the commonest causes of end-stage kidney failure and there is at present no specific treatment targeting IgAN (3). Thus, research has focused on exploring the pathogenesis of IgAN and developing effective treatment against it. As IgA deposition in the mesangial region is a key step in the development of IgAN (4) and the etiology and pathogenesis of this disease are unclear, the pathogenesis of IgAN can be discussed from the perspective of IgA deposition in the mesangial region.

Dachshund family transcription factor 1 (DACH1) is a key component of the retinal determination gene network family that has been shown to be closely associated with organogenesis and tumorigenesis $(5,6)$. DACH1 participates in cell differentiation and proliferation in renal development (7) and decreased expression of DACH1 is associated with the progression and severity of glomerulopathy (8). DACH1 disorder has been reported in a variety of human malignancies such as breast cancer (9), ovarian cancer (10), renal carcinoma (11) and gastric cancer (12). It acts as a cell-fate determination factor that regulates cell growth and development $(13,14)$, but the mechanism and function of DACH1 dysregulation in IgAN has yet to be explored.

Abnormal cell proliferation is a sign of cancer transformation (15) and abnormal expression of genes in cancer cells can be directly involved in the regulation of cell growth and cell cycle progression $(16,17)$. Malfunctions in the cell cycle usually result in uncontrolled cell growth characteristics, allowing 
cancer cells to proliferate excessively and eventually leading to the tumorigenesis (18). DACH1 inhibits cell proliferation and enhance cell cycle arrest. For example, Chen et al (19) demonstrated that DACH1 participates in p53-mediated p21 induction and cell cycle arrest in non-small-cell lung cancer. Wu et al (10) revealed that low expression of DACH1 in breast cancer cells promotes tumor growth by downregulating Nanog and Sox2. Kalousova et al (20) confirmed that DACH1 can bind to the promoter of the cell cycle inhibitor p27 to inhibit the proliferation and cell cycle progression of insulin-producing cells. The present study explored the role of DACH1 in IgAN in terms of cell proliferation and cell cycle progression.

MicroRNAs (miRNAs) are small non-coding RNAs that consist of $\sim 22$ nucleotides (21). miRNAs can degrade target mRNAs or inhibit their translation by binding to the 3'-untranslated region (3'UTR) of the target mRNAs to negatively regulate gene expression (22). Studies have shown that miRNAs are involved in the regulation of cell proliferation, differentiation, cell cycle, apoptosis and inflammation (22-25). The present study evaluated the expression and function of DACH1 in human mesangial cells (HMCs) cultured with polymeric $\operatorname{IgA}(\mathrm{pIgA})$ that was isolated and purified from the serum of patients with $\operatorname{Ig} \mathrm{AN}$ or healthy individuals. Bioinformatics analysis was used to screen for candidate miRNAs associated with DACH1 expression. Among the predicted miRNAs, miRNA (miR)-140-3p was selected and its expression and effect on cell proliferation and cell cycle progression in $\operatorname{IgAN}$ were investigated. It was found that miR-140-3p directly suppressed the expression of DACH1 and promoted the proliferation and cell cycle progression of HMCs in IgAN. The findings of the present study may provide a theoretical basis for the study of the mechanism of IgAN.

\section{Materials and methods}

Human IgAN samples. The serum of 30 patients with IgAN (men=23; women=7; age, 20-30 years $n=19$ and $>30$ years $\mathrm{n}=11$ ) and 30 normal individuals was collected. Inclusion criteria included cases of $\lg \mathrm{A}$ nephropathy and exclusion criteria included other nephropathies. The recruitment of patients was conducted at Shanghai Tenth People's Hospital between January 2020 and April 2020. The serum collection was approved by the patients and all provided written informed consent. All blood samples (each sample $\sim 5 \mathrm{ml}$ ) were anonymized. The present study was approved by the clinical research ethics committee of Shanghai Tenth People's Hospital (approval no. SHSY-IEC-4.1/20-117-01).

Extraction of polymeric $\operatorname{IgA}(\mathrm{pIgA})$. The pIgA was isolated and purified from the serum of patients with $\operatorname{IgAN}$ or healthy individuals using a jacalin-agarose column as previously described $(26,27)$. The extracted pIgA was stored at $-80^{\circ} \mathrm{C}$ until use. The final concentration of $\mathrm{pIgA}$ was $0.5 \mathrm{mg} / \mathrm{ml}$.

Cell lines and culture. Human mesangial cells (HMCs) and 293 T (cat. no. KCB200744YJ) cells were purchased from the Conservation Genetics CAS Kunming Cell Bank. HMCs were cultured in RPMI-1640 medium (Invitrogen; Thermo Fisher Scientific, Inc.) containing 10\% fetal calf serum (Gibco; Thermo Fisher Scientific, Inc.) and $80 \mathrm{U} / \mathrm{ml}$ penicillin and
$0.1 \mathrm{mg} / \mathrm{ml}$ streptomycin (Beyotime Institute of Biotechnology). $\mathrm{HMCs}$ and $293 \mathrm{~T}$ were cultured at $37^{\circ} \mathrm{C}$ in an atmosphere of $5 \%$ $\mathrm{CO}_{2}$.

Plasmids. Lentiviral vector plasmid pCDH-CMV-MCS-puro $(\mathrm{pCDH})$ and recombinant plasmid pCDH-CMV-DACH1-puro (pDACH1) were purchased from GenScript for the transfection and production of lentiviruses. The pGL3-Control plasmid and pGL3-DACH1 3'UTR luciferase reporter plasmid were purchased from GenScript for use in detection of luciferase activity following transfection.

Lentivirus transduction and production. Lentivirus packaging vector psPAX2 $(5 \mu \mathrm{g})$ and envelope vector pMD2.G $(10 \mu \mathrm{g})$ were co-transfected with lentivirus plasmid pCDH $(5 \mu \mathrm{g})$ or pDACH1 $(5 \mu \mathrm{g})$ into $293 \mathrm{~T}$ cells using Lipofectamine $2000(50 \mu \mathrm{l})$ (Thermo Fisher Scientific, Inc.) to produce the corresponding lentivirus solution. The culture supernatant was collected 48-72 $\mathrm{h}$ after transfection. The supernatant contained the corresponding virus and was used for subsequent cell infection experiments.

Reverse transcription-quantitative $(R T-q) P C R$. Total RNA was extracted from $5 \times 10^{6}$ cells using TRIzol ${ }^{\circledR}$ reagent kit according to the manufacturer's protocol (Thermo Fisher Scientific, Inc.) and reverse transcribed into single-stranded cDNA according to the instructions of the Reverse Transcription kit (Vazyme Biotech Co., Ltd.). The expression of mRNA was detected by RT-qPCR using the Rotor-Gene Q detection system (Qiagen $\mathrm{GmbH}$ ) and the reaction volume was $20 \mu \mathrm{l}$, with the following cycling conditions: $95^{\circ} \mathrm{C}$ for $5 \mathrm{~min}$, then 45 cycles of $95^{\circ} \mathrm{C}$ for $10 \mathrm{sec}$ and $60^{\circ} \mathrm{C}$ for $30 \mathrm{sec}$. The expression of miRNA or mRNA was calculated using the $2^{-\Delta \Delta \mathrm{Cq}}$ method (28). The mRNA expression level was normalized to that of GAPDH and the expression level of miRNA was normalized to U6 RNA. The primer sequences used were: IL-6 forward, 5'-AAC TCCTTCTCCACAAGCGCCTT-3' and reverse, 5'-GTCAAT TCGTTCTGAAGAGGTG-3'; IL-8 forward, 5'-GGTCTC ACCTCCCAACTGC-3' and reverse, 5'-TCAGCTCGAACA CTTTGAATAT-3'; IL-13 forward, 5'-TGAGGAGCTGGT CAACATCA-3' and reverse, 5'-CCACCTCGATTTTGGTGT CT-3'; chemokine cc-motif ligand 1 (CXCL1) forward, 5'-CTC CTGCGAGTGGCACTGCTGCTC-3' and reverse, 5'-GAG GCAAGCTTTCCGCCCATTCTT-3'; GAPDH forward, 5'-GAGTCAACGGATTTGGTCGT-3' and reverse, 5'-GAT CTCGCTCCTGGAAGATG-3'; and U6 forward, 5'-CTTCGG CAGCACATATAC-3' and reverse, 5'-TTCACGAATTTGCGT GTCAT-3'. The experiment was repeated three times.

Western blot analysis. The total protein of each group was harvested and collected using RIPA lysis buffer (Thermo Fisher Scientific, Inc.). Protein concentration was measured with a BCA Protein Assay kit (Beyotime Institute of Biotechnology). Equal amounts of protein (30 $\mu \mathrm{g} /$ lane) were separated by $10 \%$ sodium dodecyl sulfate-polyacrylamide gel electrophoresis and transferred to polyvinylidene fluoride membranes (EMD Millipore). The membrane was blocked by incubation with $5 \%$ non-fat milk for $1 \mathrm{~h}$ at $24^{\circ} \mathrm{C}$ and then with primary antibodies overnight at $4^{\circ} \mathrm{C}$. The primary antibodies used were: Anti-DACH1 (cat. no. ab176718; Abcam; 1:1,000), 
anti-Cyclin D1 (cat. no. 55506; Cell Signaling Technology, Inc.; 1:1,000), anti-Cyclin A (cat. no. 91500; Cell Signaling Technology, Inc.; 1:1,000) and anti-P21 (cat. no. 2947; Cell Signaling Technology, Inc.; 1:1,000), anti-P53 (cat. no. 2527; Cell Signaling Technology, Inc.; 1:1,000). After washing three times with Tris buffered saline-Tween (TBS-T; $50 \mathrm{mM}$ Tris, $150 \mathrm{mM} \mathrm{NaCl}, 0.05 \%$ Tween-20) the membranes were incubated with goat anti-rabbit (cat. no. ab6721; Abcam; 1:5,000) or anti-mouse (cat. no. ab205719; Abcam; 1:5,000) IgG/horseradish peroxidase secondary antibody at room temperature for $1 \mathrm{~h}$. Protein expression was detected using a chemiluminescence detection system (Tanon 4600SF; Tanon Science and Technology Co., Ltd.).

Cell proliferation assay. HMCs of each group were seeded into 96 well plates at $1 \times 10^{4}$ cells/well one day prior to cell viability measurement at day 1 , with additional measurements taken on days 3 and 5. Cell proliferation assay was determined using the CCK-8 cell count kit (cat. no. E606335, Sangon Biotech Co., Ltd.). According to the manufacturer's instructions, CCK- 8 was mixed with DMEM at 1:10 v/v, then added to 96 well plates and incubated at $37^{\circ} \mathrm{C}$ for $2 \mathrm{~h}$. The optical density (OD) values were measured at a wavelength of $562 \mathrm{~nm}$ using a multimode reader (BioTek Instruments, Inc.)

Flow cytometry. Cell cycle distribution was detected by Annexin/propidium iodide (PI) single staining (cat. no. P4170, Sigma-Aldrich; Merck KGaA). HMCs of each group were simultaneously inoculated into 6-well plates at $2 \times 10^{5}$ cells/well. After $72 \mathrm{~h}$, the cells were collected and detached with $0.25 \%$ trypsin solution. The concentration of cells was adjusted to $1 \times 10^{6}$ cells $/ \mathrm{ml}$, with $1 \mathrm{ml}$ of cells then centrifuged at $500 \mathrm{x} \mathrm{g}$ for $10 \mathrm{~min}$ at $4^{\circ} \mathrm{C}$. The supernatant was discarded and the cells were collected. The cell suspension $(1 \mathrm{ml})$ was added to $2 \mathrm{ml}$ of PBS. This was regarded as the template for further use. The cells were centrifuged $\left(500 \mathrm{x} \mathrm{g}, 10 \mathrm{~min}, 4^{\circ} \mathrm{C}\right)$ again and the supernatant removed. Next, the cells were reacted with precooled $70 \%$ (v/v) ethanol solution at $4^{\circ} \mathrm{C}$ overnight. The fixed cells were rinsed twice with PBS and then $100 \mu l$ of the cell suspension containing at least $1 \times 10^{6}$ cells $/ \mathrm{ml}$ was stained with $1 \mathrm{ml}$ of $50 \mathrm{mg} / \mathrm{l}$ PI dye solution containing RNAase $(20 \mu \mathrm{g} / \mathrm{ml})$ for $30 \mathrm{~min}$ at $25^{\circ} \mathrm{C}$ in darkness and then filtered. The cell cycle was detected by red fluorescence at an excitation wavelength of $488 \mathrm{~nm}$ and recorded using a flow cytometer (BD AccuriC6; BD Accuri C6 Software; BD Biosciences).

Enzyme-linked immunosorbent assay (ELISA). HMCs of each group were simultaneously seeded into 6-well plates at $2 \times 10^{5}$ cells/well. After $72 \mathrm{~h}$, the levels of several inflammatory cytokines including CXCL1 (cat. no. AD10945Hu), IL-6 (cat. no. AD11099Hu), IL-8 (cat. no. AD11098Hu) and IL-13 (cat. no. AD11110Hu) in HMC supernatants were determined using ELISA kits (Andy Gene Biotechnology Co., Ltd.) according to the manufacturer's instructions.

Luciferase reporter assay. miR-140-3p mimic, miR-140-3p mutant, other miRNAs and negative control was co-transfected into 293T cells together with DACH1 3'UTR luciferase reporter plasmid (GenScript). After $28 \mathrm{~h}$ of transfection, the cells were harvested using Dual-Lumi ${ }^{\mathrm{TM}}$ II Luciferase Reporter Assay kit (Beyotime Institute of Biotechnology) according to the manufacturer's instructions and the luciferase activity was detected using a multimode reader (BioTek Instruments, Inc.). The Firefly luciferase activity was normalized to the Renilla luciferase activity.

Target prediction. miRTar (miRTar, developed by Dr. Hsien-Da Huang, http://mirtar.mbc.nctu.edu.tw/human/) was used to predict binding sequences of miR-140-3p in the 3 'UTR of DACH1.

miRNA mimics, mutation and inhibition. Synthetic miR-140-3p mimic, mutation, inhibitor, other miRNAs and negative control were obtained from GenScript. The miR-140-3p mutant sequence was 5'-UACCACAGGGUAGAACCACGG-3', the miR-140-3p mutant sequence was 5'-AUGCACAGGGUAGAA CCACGG-3' and the negative control sequence was 5'-UUC UCCGAACGUGUCACGUTT-3'. RT-qPCR results confirmed that miR-140-3p mimic, mutation and inhibitor were successfully transfected into HMCs or 293 (Fig. S1).

Statistical analysis. Quantitative data are presented as the mean \pm standard deviation. The Tukey multiple comparisons test was used for statistical analysis, a one-way ANOVA was performed before Tukey's test. $\mathrm{P}>0.05$ was considered no statistical significance (ns), $\mathrm{P}<0.05$ was considered to indicate a statistically significant difference. All graphs were generated using GraphPad Prism 8.0 (GraphPad Software, Inc.). The experiment was repeated three times.

\section{Results}

Exogenous DACH1 suppresses the proliferation and altered the cell cycle of HMCs. A previous study has shown that DACH1 is expressed at low levels in IgAN and is associated with the progression and severity of glomerulopathy (8). However, its role in the development of human IgAN remains to be elucidated. The endogenous expression of DACH1 in HMCs that were cultured with pIgA-IgAN or pIgA-control was first detected. As shown in Figs. 1A and S2A, the endogenous expression of DACH1 was significantly lower in HMCs cultured with pIgA-IgAN compared with cells cultured with pIgA-control. To investigate the role of DACH1 in IgAN, HMCs were transfected with lentiviral DACH1 vectors. DACH1-transfected HMCs overexpressed DACH1 at the mRNA (Fig. S2B) and protein levels (Fig. 1B). The effect of DACH1 on cell proliferation and cell cycle progression was then examined. The cell proliferation assay demonstrated that DACH1 overexpression reversed the ability of $\mathrm{pIgA}-\operatorname{IgAN}$ to promote HMC proliferation (Fig. 1C). The cell cycle assay demonstrated that DACH1 enhanced $\mathrm{PIgA}-\mathrm{IgAN}$-induced G2 phase arrest in HMCs (Fig. 1D and E). The expression of proteins closely associated with the cell cycle were detected by western blot. As shown in Fig. 1F and G, the expression levels of Cyclin D1 and Cyclin A were upregulated in the presence of $\mathrm{pIgA}-\mathrm{IgAN}$ but were downregulated by DACH1 and the expression levels of $\mathrm{p} 21$ and $\mathrm{p} 53$ were downregulated in the presence of $\operatorname{PIgA}-\mathrm{IgAN}$ but were upregulated by DACH1. 
A

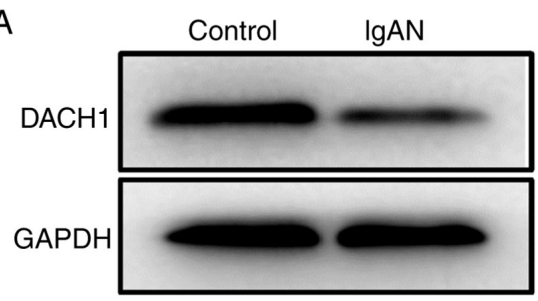

C

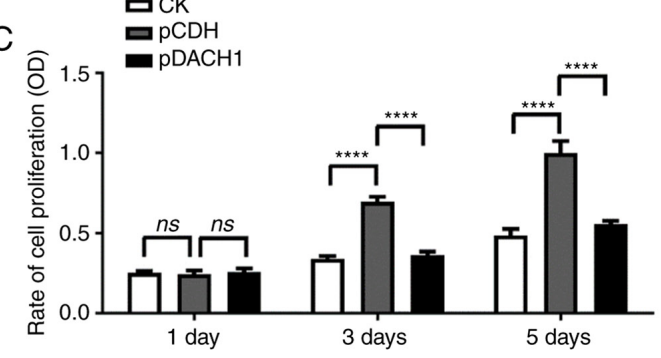

E

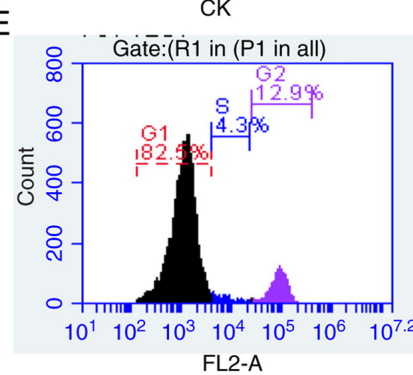

B

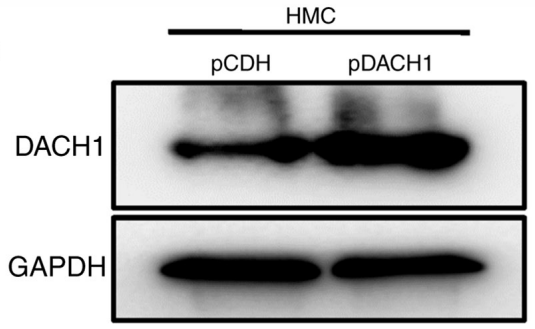

D

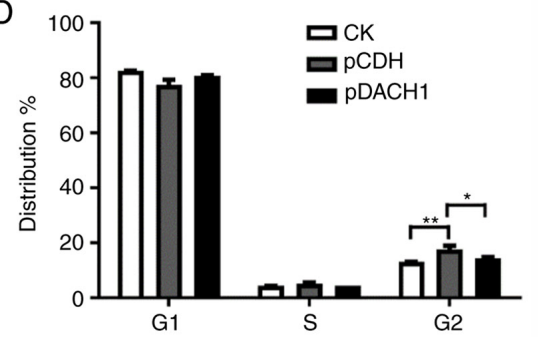

pDACH1
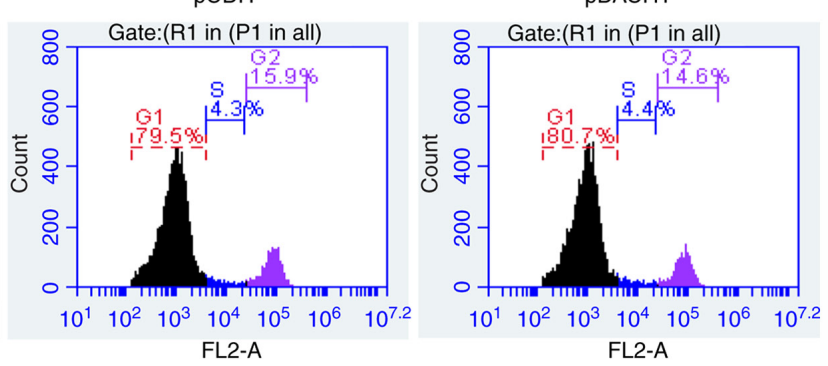

F

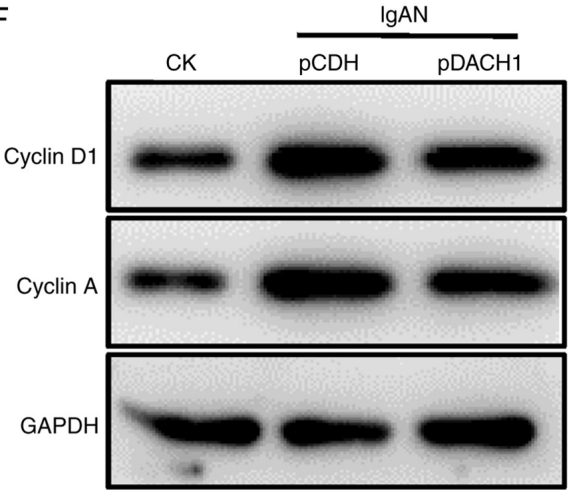

G

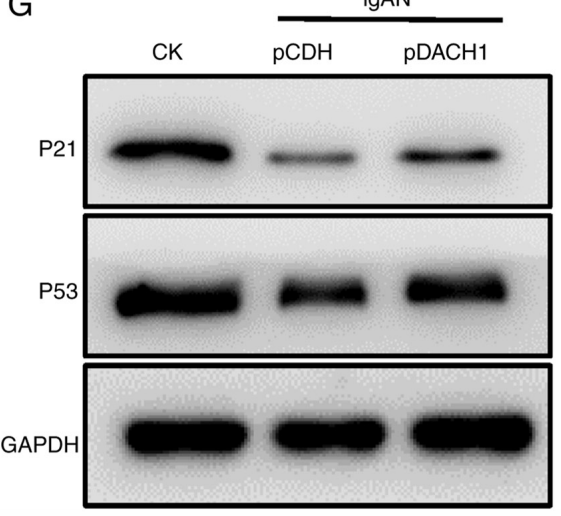

Figure 1. Overexpression of DACH1 reduces HMCs proliferation and inhibits cell cycle progression. (A) The expression of DACH1 in HMCs cultured with pIgA-IgAN or pIgA-control was detected by western blotting. (B) Western blotting for DACH1 protein in HMCs infected with DACH1 or control lentivirus (pCDH). (C) HMCs stably transduced with lentiviral DACH1, as well as the control empty vector (pCDH) were seeded and examined for cell proliferation with CCK-8 test on days 1,3 and $5\left({ }^{* * * *} \mathrm{P}<0.0001\right)$. Cell cycle assay was determined by flow cytometry. Cell cycle distribution by flow cytometry (E) and statistical results of cell cycle progression analysis (D) ( $\mathrm{P}<0.05$ and $\left.{ }^{* * *} \mathrm{P}<0.01\right)$. Protein expression of (F) Cyclin D1 and Cyclin A and (G) p21 and p53 in HMCs transduced with $\mathrm{DACH} 1$ or $\mathrm{pCDH}$ was determined by western blotting. Data are expressed as mean \pm standard deviation. DACH1, dachshund family transcription factor 1; HMCs, human mesangial cells; pIgA, polymeric IgA; IgAN, immunoglobulin A nephropathy; pCDH, pCDH-CMV-MCS-puro; ns, not significant; pDACH1, pCDH-CMV-DACH1-puro.

Exogenous DACH1 regulates inflammatory response in HMCs. To further investigate the function of DACH1 in IgAN, the release of inflammatory cytokines from HMCs that were stably transfected with DACH1 was measured. The expression of inflammatory factors (IL-6, IL-8, IL-13 and CXCL1) in the supernatant of DACH1-transfected HMCs was detected by ELISA. As shown in Fig. 2A, the concentrations of IL-6, IL-8, IL-13 and CXCL1 in HMCs cultured with PIgA-IgAN were higher compared with the control group, whereas DACH1 reversed this phenomenon by reducing the $\mathrm{PIgA}-\mathrm{IgAN}$-induced expression of IL-6, IL-8, IL-13 and CXCL1. In addition, the mRNA expression levels of IL-6, IL-8, IL-13 and CXCL1 in HMCs incubated with $0.5 \mathrm{mg} / \mathrm{ml} \mathrm{pIgA}-\operatorname{IgAN}$ were higher compared with the control group. Similarly, DACH1 downregulated the expression of IL-6, IL-8, IL-13 and CXCL1 (Fig. 2B).

Cellular miR-140-3p directly targets DACHI 3'UTR. To assess the mechanism mediating DACH1-induced inhibition of cell proliferation and inflammatory response, the present 
A
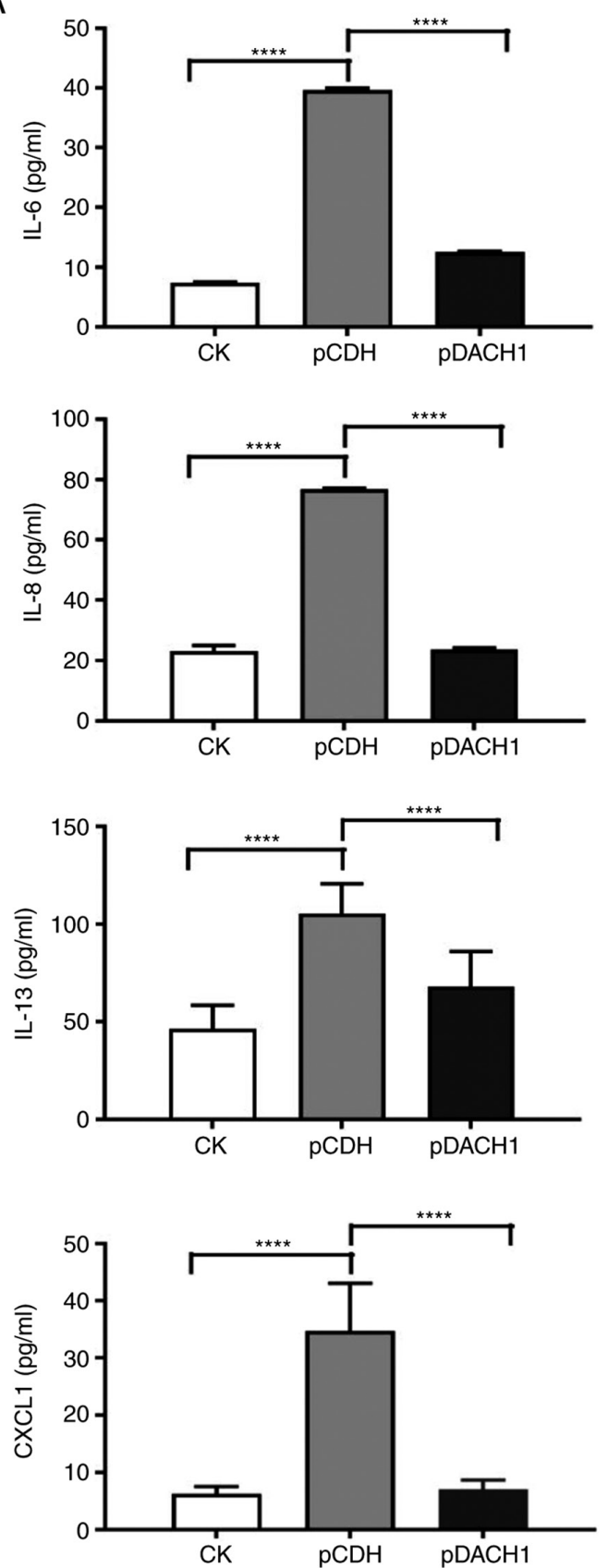

B
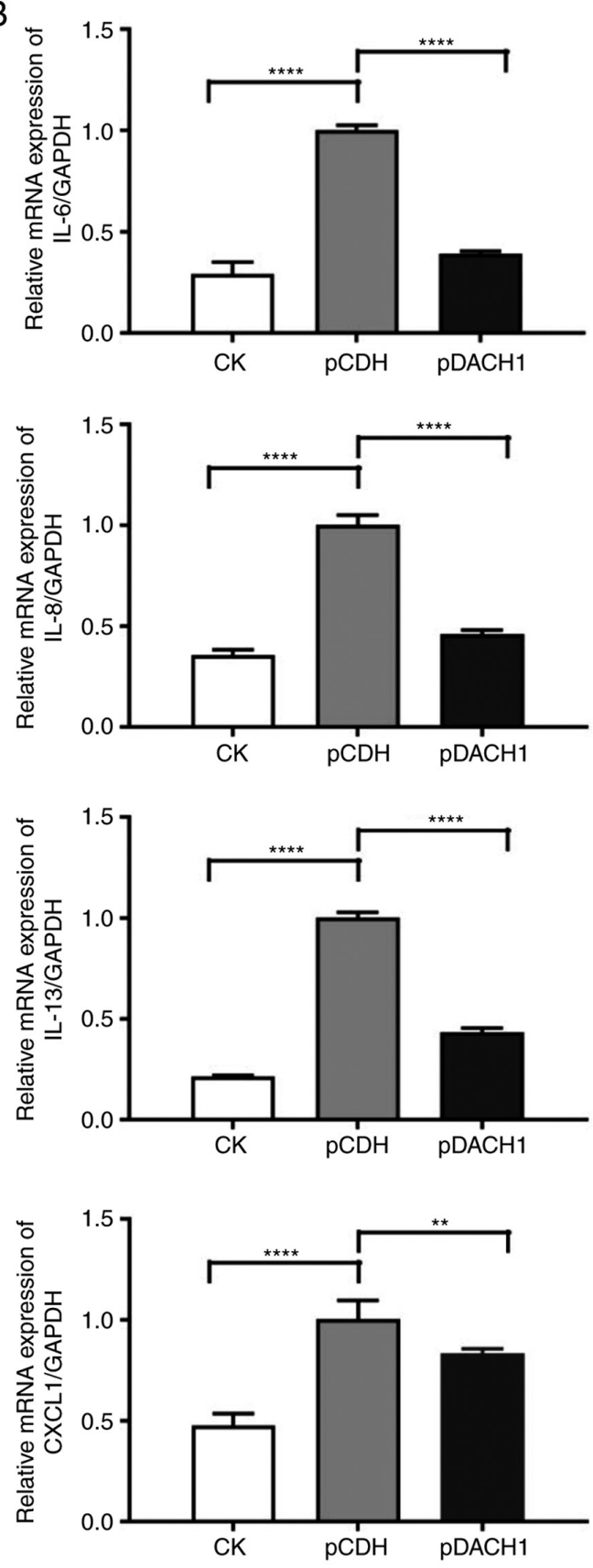

Figure 2. Overexpression of DACH1 decreases the release of inflammatory cytokines. (A) Secretion of IL-6, IL-8, IL-13 and CXCL1 (***** $<<0.0001)$ in the supernatant of HMCs transduced with the DACH1 or control (pCDH) when PIgA-IgAN was added or not. (B) Reverse transcription-quantitative PCR analyses of the mRNA levels of IL-6, IL-8, IL-13 and CXCL1 ( ${ }^{* *} \mathrm{P}<0.01$ and $\left.{ }^{* * * *} \mathrm{P}<0.0001\right)$ in HMCs transduced with the DACH1 or control (pCDH) when pIgA-IgAN was added or not. Data are expressed as mean \pm standard deviation. pCDH, pCDH-CMV-MCS-puro; ns, not significant; pDACH1, pCDH-CMV-DACH1-puro; pIgA, polymeric IgA; IgAN, immunoglobulin A nephropathy; HMCs, human mesangial cells; DACH1, dachshund family transcription factor 1.

study screened for miRNAs that may interact with DACH1 using bioinformatics software. A total of 10 miRNAs were selected, synthesized and transfected into HMCs. The mRNA and protein expression of DACH1 were detected by RT-qPCR and western blot, respectively. As shown in Figs. 3A and S2C, when miR-140-3p was transfected into HMCs, the mRNA and protein expression of DACH1 was decreased. To further verify whether DACH1 is regulated by miR-140-3p, the luciferase reporter plasmids containing the full-length sequence of DACH1 3'UTR region with 10 miRNAs we co-transfected into 293 cells. In the luciferase reporter assay, miR-140-3p reduced the luciferase activity of the 3'UTR of DACH1 (Fig. 3B). Subsequently, miR-140-3p mimics were transfected into HMCs and the expression of DACH1 detected by RT-qPCR and western blot. As shown in Fig. 3C, the expression of DACH1 was decreased by increasing the expression of miR-140-3p in a dose-dependent manner. miR-140-3p mimics and mutants were then transfected into 293 cells to examine the expression of DACH1. As shown in Fig. 3E-G, miR-140-3p mimics inhibited the expression of DACH1 and the luciferase activity of the 3'UTR of DACH1, while the miR-140-3p mutant had no 


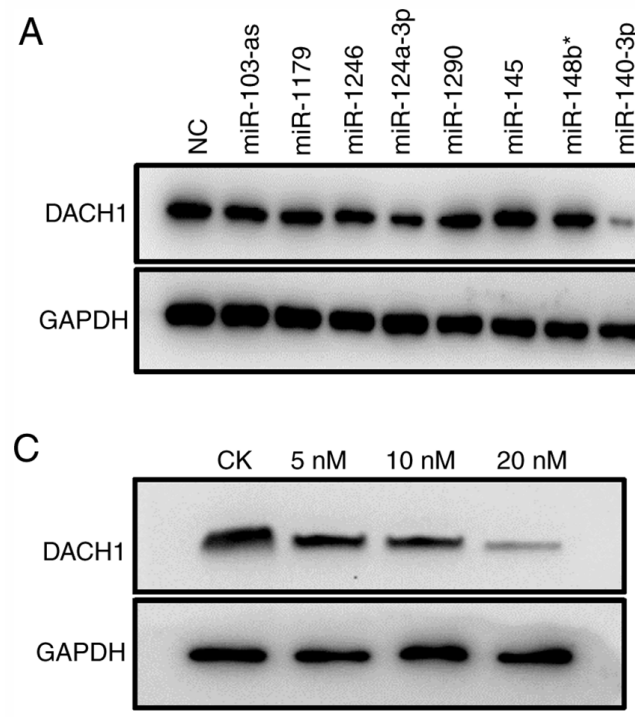

$\mathrm{B}$
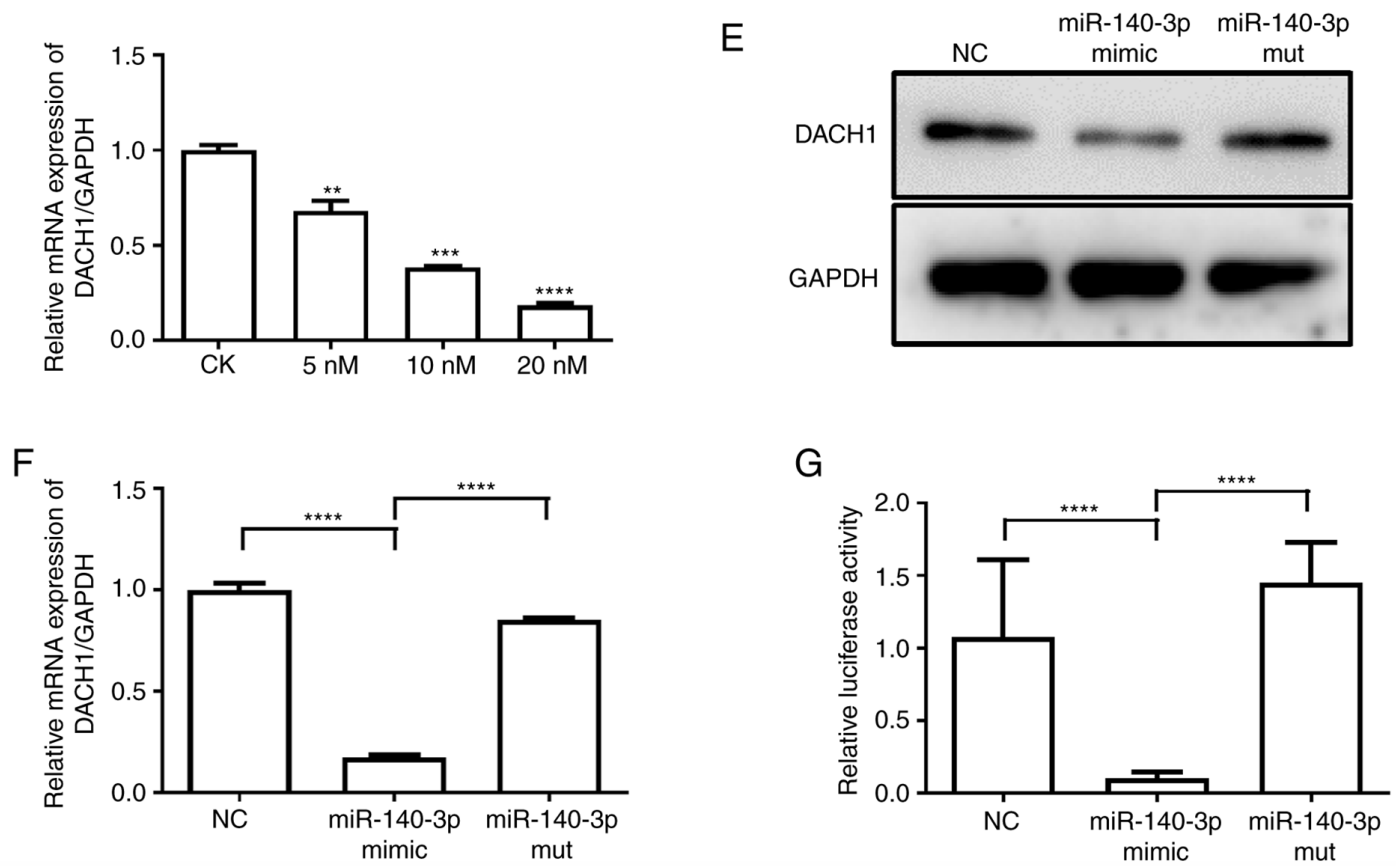

Figure 3. miR-140-3p directly targets DACH1. (A) The effect of miRNAs on endogenous DACH1 expression. HMCs were transfected with 10 miRNA mimics $(20 \mathrm{nM})$ and $\mathrm{NC}$, western blotting was performed at $48 \mathrm{~h}$ after transfection. (B) Inhibition of DACH1 3'UTR reporter activity by miRNAs. Luciferase activity was detected in 293T cells co-transfected with 10 miRNA mimics $(20 \mathrm{nM})$ or NC together with the pGL3-DACH1 3'UTR luciferase reporter $\left(^{* * * *} \mathrm{P}<0.001 \mathrm{NC}\right.$ vs. miR-140-3p). (C) miR-140-3p inhibited expression of DACH1 in a dose-dependent manner. HMCs transfected with an increasing amount (5-20 nM) of miR-140-3p mimic or NC for $48 \mathrm{~h}$. The transfected cells were collected for examined by western blotting and RT-qPCR ( ${ }^{* *} \mathrm{P}<0.01 \mathrm{NC}$ vs. $5 \mathrm{nM}$ miR-140-3p, ${ }^{* * * * *} \mathrm{P}<0.001 \mathrm{NC}$ vs. $10 \mathrm{nM}$ miR-140-3p, ${ }^{* * * * *} \mathrm{P}<0.0001 \mathrm{NC}$ vs. $20 \mathrm{nM}$ miR-140-3p). (D) Predicted binding sequences of miR-140-3p in the 3'UTR of DACH1 (miRTar, http://mirtar.mbc.nctu.edu.tw/human/). (E and F) Effect of mutation of miR-140-3p on endogenous DACH1 expression. miR-140-3p mimics and mutant were transfected into HMCs for $48 \mathrm{~h}$, while the expression of DACH1 was detected by western blotting and RT-qPCR $\left(^{* * * *} \mathrm{P}<0.0001\right)$. (G) miR-140-3p mimics/mutant or NC were co-transfected with pGL3-DACH1 3'UTR luciferase reporter into 293T cells for 48 h and the luciferase activity detected $\left({ }^{* * * *} \mathrm{P}<0.0001\right)$. Data are expressed as mean \pm standard deviation. CK, control check; miR, microRNA; DACH1, dachshund family transcription factor 1 ; HMCs, human mesangial cells; NC, negative control; 3'UTR, 3'-untranslated region; RT-qPCR, reverse transcription-quantitative PCR; mut, mutant.

effect. These data suggested that DACH1 is a direct target of, and is regulated by, miR-140-3p.

miR-140-3p inhibition reverses the effect of $\mathrm{pIgA-IgAN}$ induced cell proliferation and cell cycle changes. Next, cell proliferation and cell cycle assays were performed to determine the functional role of miR-140-3p in IgAN. First, the expression of miR-140-3p was detected in HMCs that were cultured with pIgA-IgAN or pIgA-control. As shown in
Fig. 4A, the expression of miR-140-3p was significantly higher in HMCs cultured with pIgA-IgAN compared with cells cultured with pIgA-control. Cell proliferation and cell cycle assays were performed in HMCs transfected with miR-140-3p inhibitors or negative controls in the presence of $\mathrm{pIgA}-\operatorname{Ig} \mathrm{AN}$. The cell cycle assay demonstrated that miR-140-3p enhanced pIgA-IgAN-induced $\mathrm{G}_{2}$ phase arrest and inhibited $\mathrm{G}_{1}$ phase activation in HMCs (Fig. 4B and C). The cell proliferation assay revealed that inhibition of miR-140-3p reversed the ability of 

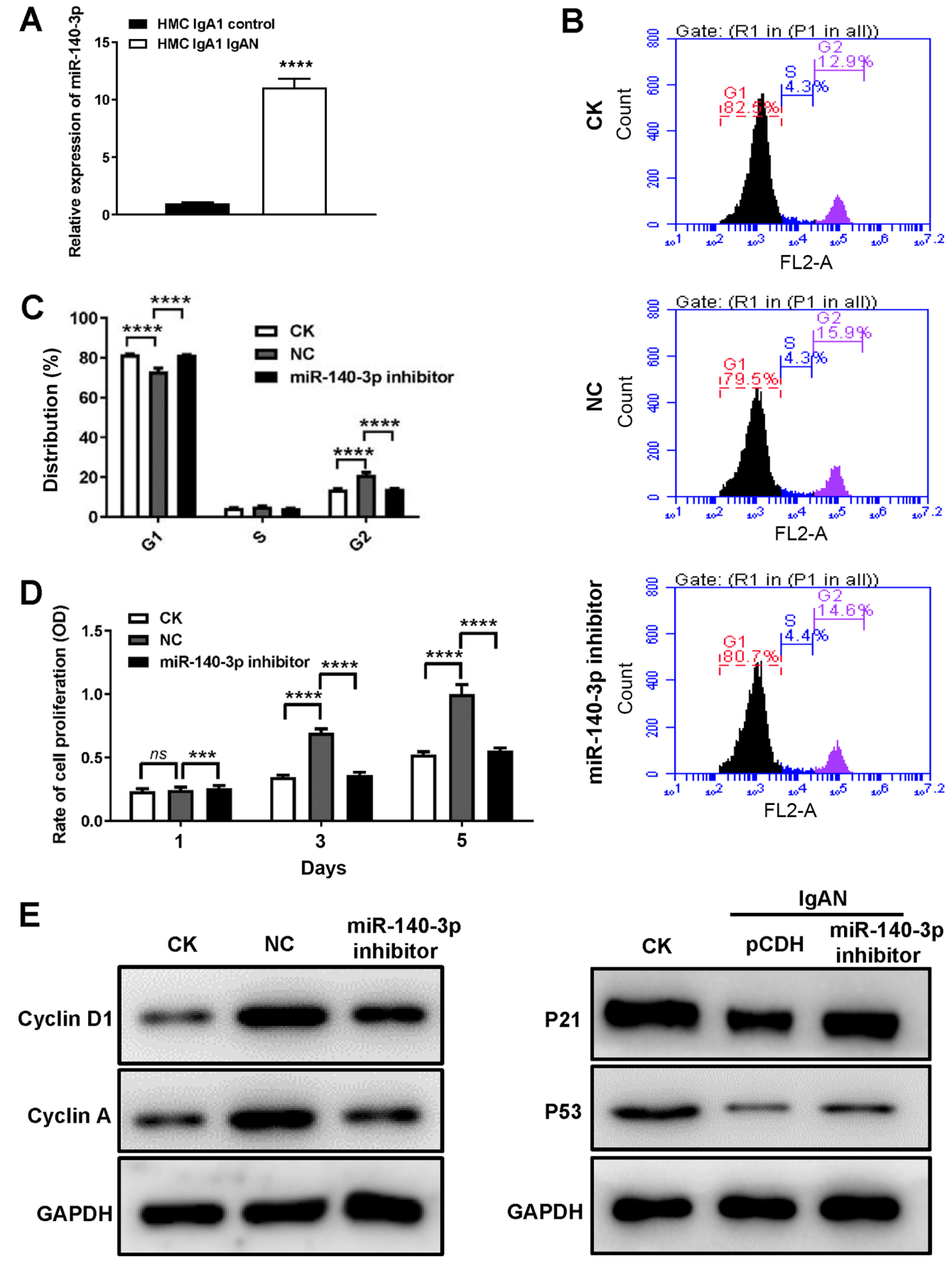

Figure 4. DACH1 decrease IgAN cell proliferation and cell cycle progression by regulating miR-140-3p expression. (A) Expression of miR-140-3p in HMCs incubated with $\mathrm{pIgA}$ or control was detected by reverse transcription-quantitative PCR ${ }^{* * * * *} \mathrm{P}<0.0001$ control vs. IgAN). Representative (B) flow cytometric graphs and (C) statistical analysis showing the distribution of cell cycle in HMCs transfected with miR-140-3p inhibitor or NC. (D) HMCs transfected with miR-140-3p inhibitor or NC were seeded and examined for cell proliferation with CCK-8 test on days 1, 3 and 5. (E) Protein expression of Cyclin D1, Cyclin A, p21 and p53 in HMCs transfected with miR-140-3p inhibitor or NC was analyzed by western blotting. Data are expressed as mean \pm standard deviation. ${ }^{* * * *} \mathrm{P}<0.001$ and ${ }^{* * * *} \mathrm{P}<0.0001$ by multiple comparisons test. CK, control check; DACH1, dachshund family transcription factor 1; IgAN, immunoglobulin A nephropathy; miR, microRNA; HMCs, human mesangial cells; NC, negative control; ns, not significant.

pIgA-IgAN to promote HMC proliferation (Fig. 4D). Upon miR-140-3p inhibition, cyclin D1 and cyclin A were downregulated whereas p21 and p53 were upregulated (Fig. 4E).

miR-140-3p also regulates inflammatory response in HMCs. To further evaluate the function of miR-140-3p in IgAN, the release of inflammatory cytokines from HMCs transfected with miR-140-3p inhibitors was detected. The supernatant was collected after transfection and the expression of

B
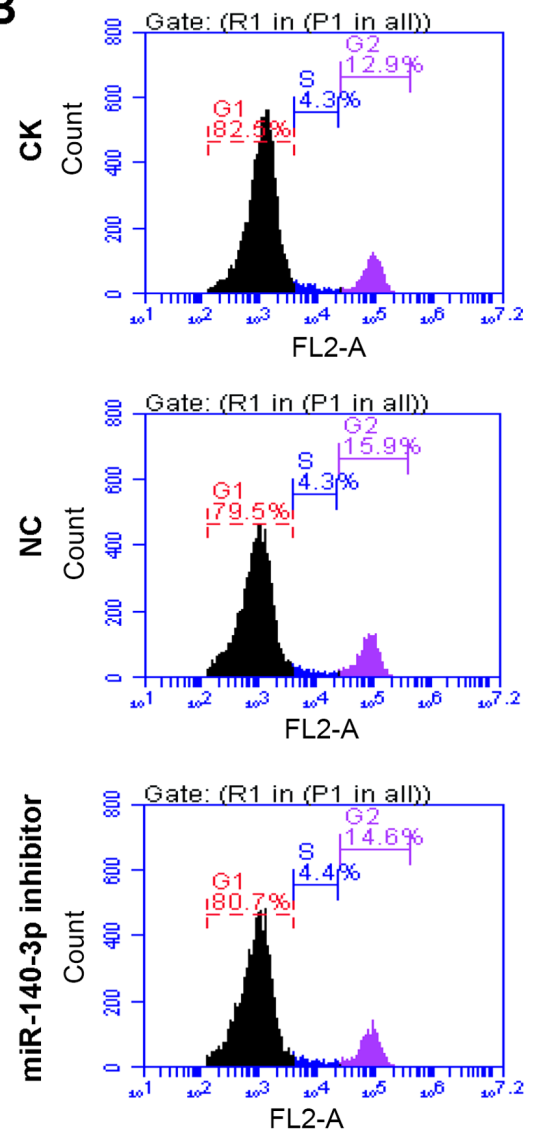

$\lg \mathrm{AN}$ 
A
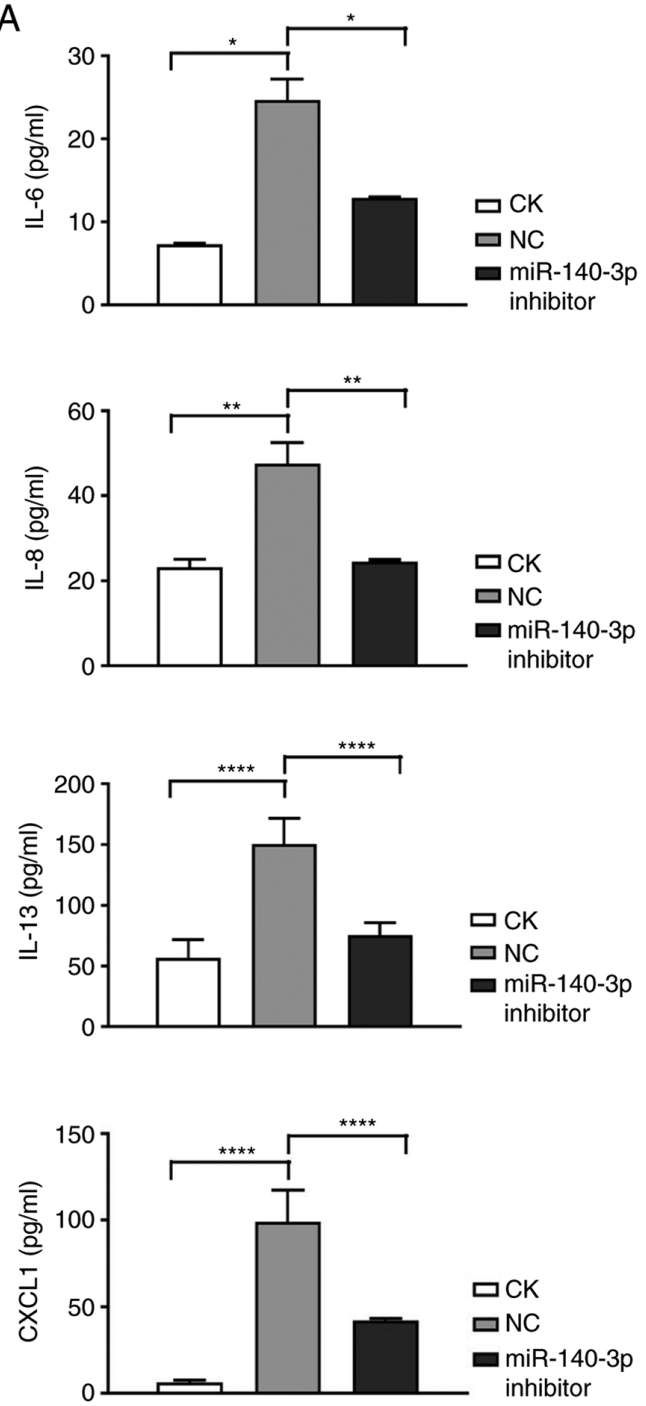

$\mathrm{B}$
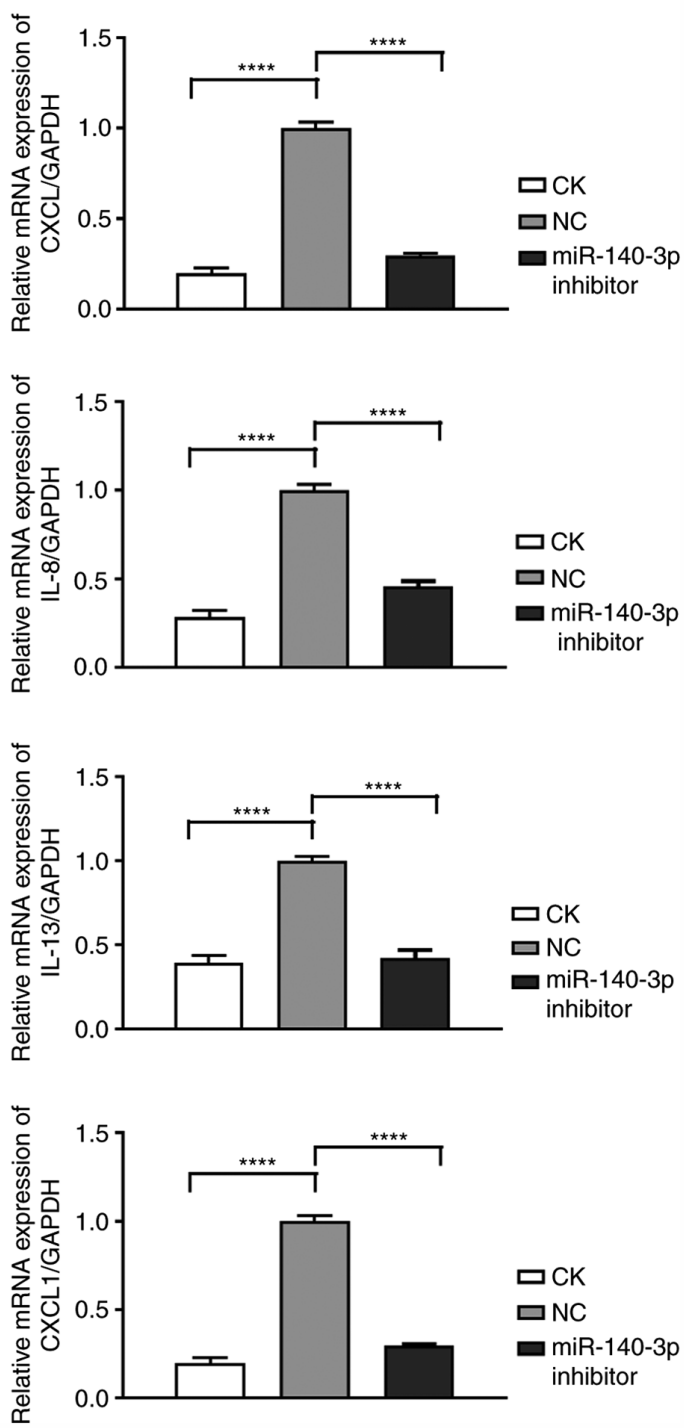

Figure 5. DACH1 decreases the release of inflammatory cytokines by positively regulating miRNA-140-3p expression. (A) Secretion of IL-6, IL-8, IL-13 and CXCL1 in the supernatant of HMCs transfected with the miR-140-3p inhibitor or NC when pIgA-IgAN was added or not. (B) Reverse transcription-quantitative PCR analyses of the mRNA levels of IL-6, IL-8, IL-13 and CXCL1 in HMCs transfected with the miR-140-3p inhibitor or NC when pIgA-IgAN was added or not. Data are expressed as mean \pm standard deviation. ${ }^{*} \mathrm{P}<0.05,{ }^{* *} \mathrm{P}<0.01$ and ${ }^{* * * *} \mathrm{P}<0.0001$. CK, control check; DACH1, dachshund family transcription factor 1; miR, microRNA; NC, negative control; HMCs, human mesangial cells; pIgA, polymeric IgA.

progress to end-stage nephropathy within 20 years of disease onset, severely affecting the physical and mental health of patients (30). A typical feature of $\operatorname{IgAN}$ is $\operatorname{Ig} \mathrm{A}$ immune complex-mediated mesangial cell proliferation and the detection of $\operatorname{Ig} \mathrm{A}$ deposition in mesangial cells by renal biopsy and immunohistochemistry is a method of $\operatorname{IgAN}$ diagnosis $(31,32)$. The pathogenesis of $\operatorname{Ig} \mathrm{AN}$ is unclear and it is important to explore the mechanism of IgAN.

DACH1 has been shown to act as a tumor suppressor gene in various malignancies (9-12) and participates in tumor cell proliferation and metabolism by regulating cell cycle-related proteins (20). Liu et al (8) suggested that DACH1 expression is decreased in IgAN and that DACH1 is involved in disease progression and severity by regulating cell cycle-related proteins. The present study hypothesized that DACH1 may be involved in regulating the cell cycle and proliferation of mesangial cells. In the present study, pIgA was isolated and purified from 30 patients with IgAN and 30 healthy individuals.
HMCs were incubated with pIgA to simulate the cell model of $\operatorname{IgA}$ deposition in mesangial cells in $\operatorname{IgAN}(27,33)$. It was found that DACH1 was downregulated in HMCs incubated with $\mathrm{pIgA}$ isolated from patients with IgAN compared with that in cells cultured with pIgA isolated from healthy individuals. In addition, exogenous DACH1 inhibited the proliferation and enhanced cell cycle arrest in HMCs induced by pIgA from patients with $\operatorname{Ig} \mathrm{AN}$.

Each phase of the cell cycle is controlled by specific regulatory proteins. Cyclin D1 mainly promotes $\mathrm{G}_{1}-\mathrm{S}$ phase transition and regulates the $\mathrm{G}_{0}$ phase reentry of stationary cells into the cell cycle in $G_{1}(34,35)$. Cyclin $A$ is essential for DNA synthesis and its expression peaks at the late $G_{1}$ phase and $\mathrm{S}$ phase $(36,37)$. p53 activation initiates cell cycle arrest at the $\mathrm{G}_{1} / \mathrm{S}$ phase (38) and p21 is involved in the regulation of $\mathrm{G}_{2} / \mathrm{M}$ transition (39). In the present study, DACH1 overexpression decreased the expression of cyclin D1 and cyclin A in HMCs that were upregulated by $\operatorname{IgA}$ from patients with $\operatorname{IgAN}$. 
According to previous study, abnormal miRNA expression is present in most patients with IgAN and is closely associated with the extent of the disease (40). miRNAs participate in almost every process involved in cancer occurrence, development and progression $(22,41-43)$. The present study confirmed that miR-140-3p expression was upregulated in HMCs treated with pIgA-IgAN and that the upregulation of miR-140-3p meditated the expression of DACH1. The present study also demonstrated that miR-140-3p regulated cell growth, cell cycle progression and the release of inflammatory cytokines in HMCs. Although the findings of the present study indicated that DACH1 was probably involved in the regulation of $\operatorname{IgAN}$ and was regulated by miR-140-3p, a variety of other factors are involved in regulating the occurrence and development of IgAN and further studies on the pathogenesis of IgAN are warranted.

In summary, the present study demonstrated that DACH1 was downregulated in HMCs in the presence of pIgA-IgAN. Moreover, DACH1 regulated cell proliferation, cell cycle progression and the release of inflammatory cytokines in HMCs and these phenomena may be targeted by miR-140-3p. This novel pathway is therefore expected to provide a weak theoretical basis for IgAN research.

\section{Acknowledgements}

Not applicable.

\section{Funding}

No funding was received.

\section{Availability of data and materials}

All data generated or analyzed during this study are included in this published article.

\section{Authors' contributions}

$\mathrm{XZ}$ and YL designed the study and performed the experiments. $\mathrm{XZ}$ drafted the manuscript. $\mathrm{PG}$ and $\mathrm{CZ}$ were major contributors in the conception, design and reviewing of the manuscript. Based on their contributions, $\mathrm{XZ}$ was listed as the first author, while $\mathrm{CZ}$ was the author for correspondence. All authors read and approved the final manuscript.

\section{Ethics approval and consent to participate}

All patients provided written informed consent and all serum samples were anonymized. The present study was approved by the clinical research ethics committee of Shanghai Tenth People's Hospital (approval no. SHSY-IEC-4.1/20-117-01).

\section{Patient consent for publication}

Not applicable.

\section{Competing interests}

The authors declare that they have no competing interests.

\section{References}

1. Lai KN: Pathogenesis of IgA nephropathy. Nat Rev Nephrol 8: 275-283, 2012

2. McGrogan A, Franssen CF and de Vries CS: The incidence of primary glomerulonephritis worldwide: A systematic review of the literature. Nephrol Dial Transplant 26: 414-430, 2011.

3. Mestecky J, Novak J, Moldoveanu Z and Raska M: IgA nephropathy enigma. Clin Immunol 172: 72-77, 2016.

4. Boyd JK, Cheung CK, Molyneux K, Feehally J and Barratt J: An update on the pathogenesis and treatment of $\operatorname{IgA}$ nephropathy. Kidney Int 81: 833-843, 2012.

5. Zhu H, Wu K, Yan W, Hu L, Yuan J, Dong Y, Li Y, Jing K, Yang Y and Guo M: Epigenetic silencing of DACH1 induces loss of transforming growth factor- $\beta 1$ antiproliferative response in human hepatocellular carcinoma. Hepatology 58: 2012-2022, 2013.

6. Popov VM, Wu K, Zhou J, Powell MJ, Mardon G, Wang C and Pestell RG: The Dachshund gene in development and hormone-responsive tumorigenesis. Trends Endocrinol Metab 21: 41-49, 2010

7. Ikeda K, Watanabe Y, Ohto H and Kawakami K: Molecular interaction and synergistic activation of a promoter by Six, Eya, and Dach proteins mediated through CREB binding protein. Mol Cell Biol 22: 6759-6766, 2002.

8. Liu QQ, Zhou YQ, Liu HQ, Qiu WH, Liu H, Hu TY, Xu Q, Lv YM and Wu KM: Decreased DACH1 expression in glomerulopathy is associated with disease progression and severity. Oncotarget 7: 86547-86560, 2016.

9. Sunde JS, Donninger H, Wu K, Johnson ME, Pestell RG, Rose GS, Mok SC, Brady J, Bonome T and Birrer MJ: Expression profiling identifies altered expression of genes that contribute to the inhibition of transforming growth factor-beta signaling in ovarian cancer. Cancer Res 66: 8404-8412, 2006.

10. Wu K, Li A, Rao M, Liu M, Dailey V, Yang Y, Di Vizio D, Wang C, Lisanti MP, Sauter G, et al: DACH1 is a cell fate determination factor that inhibits cyclin D1 and breast tumor growth. Mol Cell Biol 26: 7116-7129, 2006

11. Dalgin GS, Drever M, Williams T, King T, DeLisi C and Liou LS: Identification of novel epigenetic markers for clear cell renal cell carcinoma. J Urol 180: 1126-1130, 2008.

12. Yamada Y, Arao T, Gotoda T, Taniguchi H, Oda I, Shirao K, Shimada Y, Hamaguchi T, Kato K, Hamano T, et al: Identification of prognostic biomarkers in gastric cancer using endoscopic biopsy samples. Cancer Sci 99: 2193-2199, 2008.

13. Chen R, Amoui M, Zhang Z and Mardon G: Dachshund and eyes absent proteins form a complex and function synergistically to induce ectopic eye development in Drosophila. Cell 91: 893-903, 1997.

14. Shen W and Mardon G: Ectopic eye development in Drosophila induced by directed dachshund expression. Development 124: 45-52, 1997.

15. Fan GK, Imanaka M, Yang B and Takenaka H: Characteristics of nasal inverted papilloma and its malignant transformation: A study of cell proliferation and programmed cell death. Am J Rhinol 20: 360-363, 2006.

16. Liu M, Zhang H, Li Y, Wang R, Li Y, Zhang H, Ren D, Liu H, Kang C and Chen J: HOTAIR, a long noncoding RNA, is a marker of abnormal cell cycle regulation in lung cancer. Cancer Sci 109: 2717-2733, 2018.

17. Wang W, Dong M, Cui J, Xu F, Yan C, Ma C, Yi L, Tang W, Dong $J$ and Wei Y: NME4 may enhance nonsmall cell lung cancer progression by overcoming cell cycle arrest and promoting cellular proliferation. Mol Med Rep 20: 1629-1636, 2019.

18. Hanahan D and Weinberg RA: Hallmarks of cancer: The next generation. Cell 144: 646-674, 2011.

19. Chen K, Wu K, Cai S, Zhang W, Zhou J, Wang J, Ertel A, Li Z, Rui H, Quong A, et al: Dachshund binds p53 to block the growth of lung adenocarcinoma cells. Cancer Res 73: 3262-3274, 2013.

20. Kalousova A, Mavropoulos A, Adams BA, Nekrep N, Li Z, Krauss S, Stainier DY and German MS: Dachshund homologues play a conserved role in islet cell development. Dev Biol 348: $143-152,2010$.

21. He L and Hannon GJ: MicroRNAs: Small RNAs with a big role in gene regulation. Nat Rev Genet 5: 522-531, 2004.

22. Bartel DP: MicroRNAs: Genomics, biogenesis, mechanism, and function. Cell 116: 281-297, 2004.

23. Kloosterman WP and Plasterk RH: The diverse functions of microRNAs in animal development and disease. Dev Cell 11: 441-450, 2006. 
24. Li K, Du Y, Jiang BL and He JF: Increased microRNA-155 and decreased microRNA-146a may promote ocular inflammation and proliferation in Graves' ophthalmopathy. Med Sci Monit 20: 639-643, 2014.

25. Wang W, Zhao LJ, Tan YX, Ren H and Qi ZT: miR-138 induces cell cycle arrest by targeting cyclin D3 in hepatocellular carcinoma. Carcinogenesis 33: 1113-1120, 2012.

26. Tamouza H, Vende F, Tiwari M, Arcos-Fajardo M, Vrtovsnik F, Benhamou M, Monteiro RC and Moura IC: Transferrin receptor engagement by polymeric IgA1 induces receptor expression and mesangial cell proliferation: Role in IgA nephropathy. Contrib Nephrol 157: 144-147, 2007.

27. Lai KN, To WY, Li PK and Leung JC: Increased binding of polymeric lambda-IgA to cultured human mesangial cells in $\operatorname{IgA}$ nephropathy. Kidney Int 49: 839-845, 1996.

28. Livak KJ and Schmittgen TD: Analysis of relative gene expression data using real-time quantitative PCR and the 2(-Delta Delta C(T)) method. Methods 25: 402-408, 2001.

29. Schena FP and Nistor I: Epidemiology of IgA nephropathy: A global perspective. Semin Nephrol 38: 435-442, 2018.

30. Tian J, Wang Y, Liu X, Zhou X and Li R: Rapamycin ameliorates IgA nephropathy via cell cycle-dependent mechanisms. Exp Biol Med (Maywood) 240: 936-945, 2015

31. Kurogi Y: Mesangial cell proliferation inhibitors for the treatment of proliferative glomerular disease. Med Res Rev 23: 15-31, 2003.

32. Rodrigues JC, Haas M and Reich HN: IgA nephropathy. Clin J Am Soc Nephrol 12: 677-686, 2017.

33. Leung JC, Chan LY, Tang SC, Lam MF, Chow CW, Lim AI and Lai KN: Oxidative damages in tubular epithelial cells in IgA nephropathy: Role of crosstalk between angiotensin II and aldosterone. J Transl Med 9: 169, 2011.
34. Morgan DO: Principles of CDK regulation. Nature 374: 131-134, 1995.

35. Kim JK and Diehl JA: Nuclear cyclin D1: An oncogenic driver in human cancer. J Cell Physiol 220: 292-296, 2009.

36. Wolf $\mathrm{G}$ and Shankland SJ: Cell cycle control in glomerular disease. Prog Cell Cycle Res 5: 71-79, 2003.

37. Shankland SJ and Wolf G: Cell cycle regulatory proteins in renal disease: Role in hypertrophy, proliferation, and apoptosis. Am J Physiol Renal Physiol 278: F515-F529, 2000.

38. Li Y, Liu Z, Guo X, Shu J, Chen Z and Li L: Aristolochic acid I-induced DNA damage and cell cycle arrest in renal tubular epithelial cells in vitro. Arch Toxicol 80: 524-532, 2006.

39. Marshall CB and Shankland SJ: Cell cycle and glomerular disease: A minireview. Nephron Exp Nephrol 102: e39-48, 2006.

40. Dai Y, Sui W, Lan H, Yan Q, Huang H and Huang Y: Microarray analysis of micro-ribonucleic acid expression in primary immunoglobulin A nephropathy. Saudi Med J 29: 1388-1393, 2008

41. Silveri L, Tilly G, Vilotte JL and Le Provost F: MicroRNA involvement in mammary gland development and breast cancer. Reprod Nutr Dev 46: 549-556, 2006.

42. Zhang B, Pan X, Cobb GP and Anderson TA: microRNAs as oncogenes and tumor suppressors. Dev Biol 302: 1-12, 2007.

43. Khoshnaw SM, Green AR, Powe DG and Ellis IO: MicroRNA involvement in the pathogenesis and management of breast cancer. J Clin Pathol 62: 422-428, 2009.

(i) (9) This work is licensed under a Creative Common cc) Attribution-NonCommercial-NoDerivatives 4.0 International (CC BY-NC-ND 4.0) License. 